\title{
Hábitos alimentares e risco de doenças cardiovasculares em universitários
}

\section{Eating habits and risk of cardiovascular disease in college students}

\author{
Gustavo A. Oliveira'; Samuel H. V. Oliveira'; Charles A. S. Morais²; Luciana M. Lima
}

\section{RESUMO}

Modelo de estudo: Pesquisa descritiva, observacional, transversal.

Objetivo: Descrever qualitativamente a frequência de ingestão de determinados alimentos, considerados de risco e de proteção para doenças cardiovasculares (DCV), além de determinar o Escore de Risco de Framingham (ERF) em indivíduos supostamente saudáveis, estudantes de graduação de uma universidade pública brasileira.

Metodologia: Participaram 97 estudantes, 45 homens e 52 mulheres, na faixa etária de 18 a 25 anos. Após assinatura do Termo de Consentimento Livre e Esclarecido (TCLE), aprovado pelo Comitê de Ética em Pesquisa, os estudantes preencheram um questionário com os dados da pesquisa. Foram estudados alimentos classificados como de risco e de proteção, conforme sua composição química avaliada por tabelas de alimentos. O teste Qui-quadrado fui utilizado quando as frequências esperadas foram iguais ou superiores a 5. Para os demais parâmetros foi utilizado o teste exato de Fischer.

Resultados: Entre os alimentos protetores destacou-se a ingestão diária de legumes (33\%), verduras $(22 \%)$ e frutas (17\%) e entre os de risco estão a ingestão diária de café/chás com açúcar (39\%), maionese/margarina/manteiga (34\%) e doces (14\%). Houve variação de consumo conforme o sexo, para as frequências de 0,1, 2 e 4 vezes por semana para os alimentos: farinha de milho/mandioca, biscoito maisena/caseiro/água e sal, aveia, frango com pele. Houve variação significativa de consumo diário entre os sexos para os alimentos: frutas, doces, maionese/margarina/manteiga, biscoito maisena/caseiro/água e sal.

Conclusões: Este estudo demonstrou que os estudantes universitários apresentaram uma maior frequência diária de ingestão de alimentos considerados de proteção para DCV do que alimentos de risco. Em adição, o ERF calculado demonstrou baixo risco de desenvolvimento de DCV nos indivíduos avaliados.

Palavras-chaves: Hábitos Alimentares. Adulto Jovem. Doenças Cardiovasculares.

1. Bolsista de Iniciação Científica, Graduando em Medicina, Departamento de Medicina e Enfermagem, Universidade Federal de Viçosa, Viçosa, Minas Gerais

2. Bolsista de Iniciação Científica, Graduando em Bioquímica, Departamento de Bioquímica e Biologia Molecular, Universidade Federal de Viçosa, Viçosa, Minas Gerais

3. Professora Adjunta, Curso de Medicina, Departamento de Medicina e Enfermagem, Universidade Federal de Viçosa, Viçosa, Minas Gerais
CORRESPONDÊNCIA:

Profa. Dra. Luciana Moreira Lima

Universidade Federal de Viçosa, Departamento de Medicina e Enfermagem Av. PH Rolfs, s/n - Centro CEP 36570-000 Viçosa, MG, Brasil

Artigo recebido em 20/03/2013 Aprovado para publicação em 28/03/2014 


\section{Introdução}

A dieta habitual parece ser um elemento fundamental de análise dos determinantes da susceptibilidade para a aterosclerose e doenças isquêmicas do coração. ${ }^{1}$

Entre os fatores de risco para tais doenças encontram-se a dieta rica em gorduras saturadas e colesterol que estão associados a valores séricos elevados de colesterol total e $\mathrm{LDL}^{2}$, consumo de bebida alcoólica ${ }^{1}$, consumo de sal, cuja diferença de $100 \mathrm{mEq}$ por dia na ingestão de $\mathrm{NaCl}$ estaria associada a uma diferença de 3 a $6 \mathrm{mmHg}$ na pressão arterial sistólica ${ }^{3}$ e dieta rica em calorias. ${ }^{1}$ Diversos estudos tem avaliado a influência da ingestão elevada de ácidos graxos trans sobre os níveis da lipoproteína(a) [Lp(a)], considerada um fator de risco para doenças cardiovasculares (DCV). Os alimentos contendo gordura parcialmente hidrogenada contribuem com cerca de $80 \%$ a $90 \%$ da ingestão diária de ácidos graxos trans. ${ }^{4}$ As dietas com baixos teores de lipídios, e, consequentemente, ricas em carboidratos, elevam significativamente os triglicerídeos sanguíneos e reduzem os níveis de HDL. ${ }^{2}$

Com relação à proteção dos alimentos alguns merecem destaque. $\mathrm{O}$ consumo de soja tem sido associado à redução de DCV, especialmente da aterosclerose em modelos animais. Em adição, evidências epidemiológicas sugerem que populações que consomem dietas ricas em soja e seus produtos apresentam uma menor taxa de mortalidade por doenças coronarianas. ${ }^{5}$ Enquanto uma dieta restrita em lipídios pode diminuir as concentrações de colesterol sérico de 5 a $10 \%$, o uso de alimentos ricos em fibra solúvel, como farelo de aveia e produtos de feijão, podem produzir uma diminuição de 20 a $30 \% .{ }^{6}$ As fibras solúveis e insolúveis têm apresentado, quando ingeridas, efeitos fisiológicos diferenciados. O efeito hipocolesterolêmico das fibras é atribuído à sua fração solúvel. Na prevenção de doenças ateroscleróticas alguns antioxidantes têm recebido maior atenção, por sua possível ação benéfica e entre estes estão as vitaminas C (ácido ascórbico) e E (tocoferol), os carotenóides e os flavonóides, que constituem o maior grupo de compostos fenólicos existentes na natureza e estão amplamente distribuídos nos vegetais. ${ }^{7}$

Burr et al. (1989) conduziram um estudo randomizado controlado, em homens, a fim de examinar os efeitos da gordura do peixe na recidiva de infarto do miocárdio. Os indivíduos recrutados eram orien- tados a consumir no mínimo duas porções de peixe semanalmente (entre 200 e $400 \mathrm{~g}$ ), enquanto o grupo controle não recebeu esse tipo de orientação. Os grupos foram acompanhados durante cerca de dois anos, onde também foram observados os níveis plasmáticos de lipídios. Após o período de seguimento, Burr et al. (1989) demonstraram uma redução de cerca de $29 \%$ da mortalidade no grupo que havia consumido a dieta com um maior conteúdo de gordura de peixe. ${ }^{8}$ Além disso, o consumo de ácidos graxos poliinsaturados e de ácidos graxos monoinsaturados tem sido recomendado para melhorar o perfil lipídico em relação aos ácidos graxos saturados. ${ }^{9}$

A composição do café e sua repercussão sobre a saúde humana, notadamente sobre as doenças cardiovasculares, vêm sendo objeto de muitos estudos, cujos resultados são conflitantes. Tal fato, provavelmente, se deve à presença de substâncias com efeitos antagônicos em potencial, ou seja, ao mesmo tempo antioxidantes e com potencial para a elevação do colesterol sérico e para o aumento agudo da pressão arterial sistêmica. ${ }^{10}$

Como o consumo de determinados alimentos pode influenciar na avaliação do risco de DCV, torna-se altamente desejável o conhecimento dos hábitos alimentares em todas as faixas etárias, bem como a estratificação do risco cardiovascular, para que estratégias de mudanças no estilo de vida possam ser instituídas numa tentativa de minimizar o risco de DCV em nossa população. A maioria dos indivíduos que apresenta DCV adquire alguns dos fatores de risco na infância e juventude, mantendo um estilo de vida pouco saudável durante a idade adulta. Estratégias que visem à mudança de hábito devem ter como público alvo crianças, adolescentes e indivíduos jovens, preferencialmente.

Em virtude dos efeitos funcionais exercidos pelos alimentos, o objetivo deste estudo foi descrever qualitativamente a frequência de ingestão de determinados alimentos considerados de risco e de proteção para DCV, além de calcular o Escore de Risco de Framingham (ERF) em indivíduos supostamente saudáveis, estudantes de graduação de uma universidade pública.

\section{Métodos}

Para avaliação dos hábitos alimentares foram entrevistados 97 estudantes de graduação, de 18 a 25 anos, de ambos os sexos, sendo 45 do sexo masculino 
e 52 do sexo feminino, ambos supostamente saudáveis, no período de Maio a Novembro de 2011. Embora alguns alunos fizessem as refeições no restaurante universitário da instituição, a simples avaliação do cardápio do mesmo não representaria da mesma forma que um questionário sobre seus hábitos alimentares, uma vez que os indivíduos poderiam não fazer ingestão do que é oferecido no cardápio e fazer outras refeições em locais diferentes.

Após assinar o termo de consentimento livre e esclarecido aprovado pelo Comitê de Ética em Pesquisa da instituição, cada estudante recebia um questionário contendo os alimentos ou pequenos grupos de alimentos que se assemelhavam e marcava, ele próprio, uma das opções de frequência de consumo semanal a seguir: 7 dias por semana, 4 dias por semana, 2 dias por semana, 1 dia por semana, ou nunca consumia determinado alimento. Os alimentos do questionário não estavam separados nem classificados como de proteção ou de risco para não influenciar na resposta do entrevistado. Os alimentos consumidos diariamente foram os utilizados para avaliação da capacidade funcional de proteção ou de risco para DCV.

Para análise foram consideradas as tabelas de alimentos usada nos Estados Unidos ${ }^{11,12}$ e no Brasil ${ }^{13,14}$ para classificar em alimentos e/ou preparações considerados de risco para DCV (fontes de gorduras saturadas e/ou colesterol e/ou ácidos graxos trans e/ ou sódio e/ou glicídios) e considerados protetores para doenças cardiovasculares (fontes de fibras, antioxidantes e ácidos graxos insaturados). ${ }^{15} \mathrm{O}$ grupo dos alimentos de risco ficou composto pelos alimentos contendo alto conteúdo de carboidratos: Arroz, Mandioca/batata/inhame, Farinha de milho/mandioca, Doces (compotas, chocolates, confeitos), Refrigerante com açúcar; Café/chás com açúcar, Pães/bolos, Biscoito recheado, Biscoito água e sal/maisena/caseiro. Também por grande conteúdo de gordura saturada e colesterol: Leite/iogurte/queijo, Maionese/margarina/ manteiga, Carne de boi, Carne/linguiça de porco, Frango com pele, Embutidos (presunto/salame/mortadela/ salsicha), Ovo, Massas (macarrão instantâneo, lasanha, ravióli). E pela grande quantidade de carboidratos, colesterol, gordura saturada, gordura trans e sódio: Pizza/hambúrguer/cachorro quente/salgadinhos fritos ou assados, sendo também possível encontrar aqui alguns pães, bolos, biscoitos recheados e os embutidos. Os alimentos que continham álcool também ingressaram no grupo de risco: Vinho tinto, Outros vinhos, cerveja, Destilados (Uísque/cachaça/vodka/rum).
Para a formação do grupo dos alimentos de proteção foram observadas as fontes de fibras solúveis e de antioxidantes: feijão, soja e seus derivados (exceto o óleo de soja), aveia, além de verduras, legumes e frutas. O conteúdo significativo de ácidos graxos insaturados está presente no azeite e no peixe. Os alimentos sem classificação foram: Refrigerante diet/ light, Café/chás com adoçante, frango sem pele.

Nenhum participante do estudo relatou diabetes ou hipertensão. A pressão arterial também foi aferida no estado de repouso. As amostras de sangue foram obtidas a partir do estudante em jejum de 12 horas, usando tubos de vácuo sem anticoagulante. Colesterol total, HDL, LDL e triglicéridos foram analisados por métodos específicos. O Escore de Risco de Framingham (ERF) foi calculado considerando a idade, colesterol total, HDL-colesterol, tabagismo, diabetes e pressão arterial sistólica.

O teste Qui-quadrado fui utilizado quando as frequências esperadas de cada célula foram iguais ou superiores a cinco. Para os demais parâmetros foi utilizado o teste exato de Fischer. O teste t de Student foi utilizado para análise estatística das variáveis paramétricas. O nível de significância adotado para o estudo foi de $5 \%$.

\section{Resultados}

A prevalência de ingestão diária de alimentos de risco e de proteção encontra-se na figura 1. Entre os alimentos considerados de proteção, a ingestão diária de legumes (33\%), verduras $(22 \%)$ e frutas $(17 \%)$ mostraram-se significativamente superiores $(\mathrm{p}<0,001)$ à ingestão diária de soja $(4 \%)$, aveia $(5 \%)$ e azeite (4\%).Entre os alimentos considerados de risco, a ingestão diária de café/chás com açúcar (39\%), maionese/margarina/manteiga (34\%) e doces (14\%) mostraram-se significativamente superiores $(\mathrm{p}<0,05)$ à ingestão diária de pizza/hambúrguer/cachorro quente/salgadinhos (5\%), refrigerante com açúcar (3\%), embutidos $(3 \%)$ e massas $(2 \%)$.

A ingestão diária de saladas (59\%), legumes (41\%), doces (24\%) e frutas (19\%) mostraram-se significativamente superior $(\mathrm{p}<0,01)$ à ingestão diária de refrigerantes com açúcar (2\%), carne de porco $(1 \%)$, ovos $(0,6 \%)$ e carne de frango (1\%) nos estudantes avaliados.

Houve variação de consumo conforme o sexo, para as frequências de 0,1, 2 e 4 vezes por semana para os alimentos: farinha de milho/mandioca, bis- 


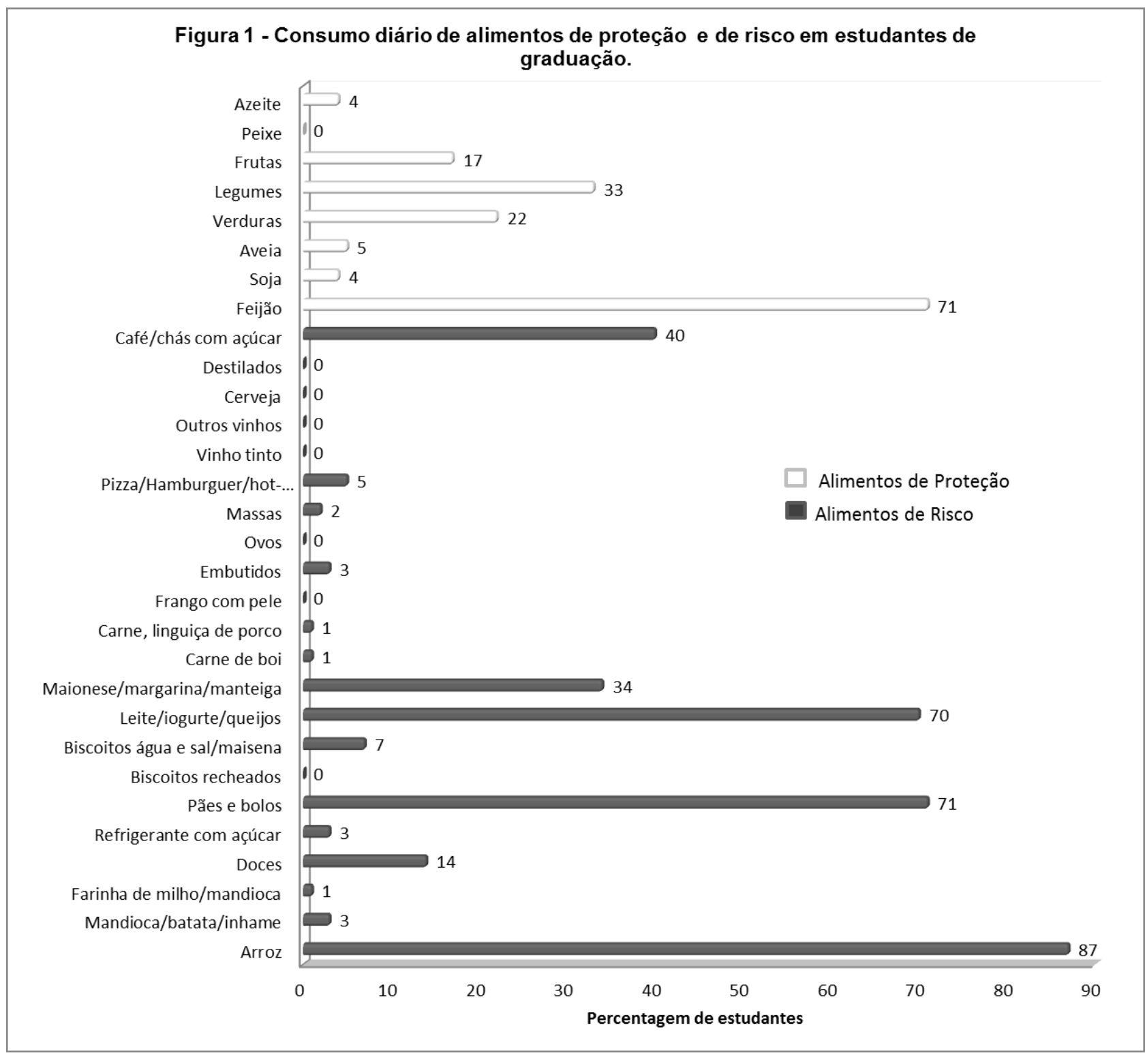

Figura 1: Consumo diário de alimentos de proteção e de risco para doenças cardiovasculares.

coito maisena/caseiro/água e sal, aveia, frango com pele, conforme mostra a tabela 1. Para os outros alimentos não houve diferença significante. Houve variação significativa de consumo diário entre os sexos para os alimentos: frutas, doces, maionese/margarina/manteiga e também para o biscoito maisena/caseiro/água e sal conforme mostra a figura 2.

Os níveis plasmáticos de colesterol total e de HDL foram significativamente menores $(\mathrm{p}<0,05)$ nos homens, quando comparadas com mulheres. Não foram observadas diferenças significativas entre homens e mulheres para triglicerídeos ou LDL. No entanto, as mulheres apresentaram uma porcentagem significativamente maior (37\%) de dislipidemia do que os homens $(17 \%, \mathrm{p}=0,015)$. Os valores médios e desvios padrão obtidos para o ERF de homens e mulheres foram $-4,0 \pm 0,6$ e $-1,7 \pm 0,3$, respectivamente, com diferença significativa observada $(\mathrm{p}=0,0002)$. Todos os participantes do estudo foram classificados no grupo de baixo risco para um evento cardiovascular nos próximos 10 anos, de acordo com os dados obtidos para o ERF. 
Tabela I - Alimentos com variação de consumo semanal conforme o sexo em estudantes de graduação

\begin{tabular}{|c|c|c|c|c|c|c|c|c|c|c|c|c|}
\hline & \multicolumn{3}{|c|}{ Nunca } & \multicolumn{3}{|c|}{ 1x/semana } & \multicolumn{3}{|c|}{$2 \mathrm{x} /$ semana } & \multicolumn{3}{|c|}{$4 \mathrm{x} /$ semana } \\
\hline & $M(n=45)$ & $\mathrm{F}(\mathrm{n}=52)$ & $\mathrm{P}$ & $M(n=45)$ & $\mathrm{F}(\mathrm{n}=52)$ & $\mathrm{p}$ & $M(n=45)$ & $\mathrm{F}(\mathrm{n}=52)$ & $\mathrm{P}$ & $\mathrm{M}(\mathrm{n}=45)$ & $\mathrm{F}(\mathrm{n}=52)$ & $\mathrm{p}$ \\
\hline $\begin{array}{l}\text { Farinha } \\
\text { de milho/ } \\
\text { mandioca }\end{array}$ & $7(16 \%)$ & $14(27 \%)$ & $n s$ & $17(38 \%)$ & $26(50 \%)$ & $n s$ & $16(36 \%)$ & $9(17 \%)$ & $0,04^{(*)}$ & $3(7 \%)$ & $3(6 \%)$ & $n s$ \\
\hline $\begin{array}{l}\text { Biscoito } \\
\text { maisena/ } \\
\text { caseiro/ } \\
\text { água e sal }\end{array}$ & $10(23 \%)$ & $10(19 \%)$ & $n s$ & $15(33 \%)$ & $7(13 \%)$ & $0,019^{(*)}$ & $13(29 \%)$ & $19(37 \%)$ & $n s$ & $6(13 \%)$ & $9(17 \%)$ & $n s$ \\
\hline Aveia & $31(69 \%)$ & $28(54 \%)$ & $n s$ & $3(7 \%)$ & $11(21 \%)$ & $n s$ & $7(16 \%)$ & $5(10 \%)$ & $n s$ & 0 & $6(12 \%)$ & $0,02^{(* *)}$ \\
\hline $\begin{array}{l}\text { Frango } \\
\text { com pele }\end{array}$ & $14(31 \%)$ & $28(54 \%)$ & $0,024^{(*)}$ & $16(36 \%)$ & $16(31 \%)$ & $n s$ & $13(29 \%)$ & $4(8 \%)$ & $0,006^{(* *)}$ & $1(2 \%)$ & $4(8 \%)$ & $n s$ \\
\hline
\end{tabular}

$n s=$ não significativo, $\mathrm{M}$ = sexo masculino, $\mathrm{F}=$ sexo feminino, $\left({ }^{*}\right)$ Qui-quadrado, $\left({ }^{* *}\right)$ Teste Exato de Fisher

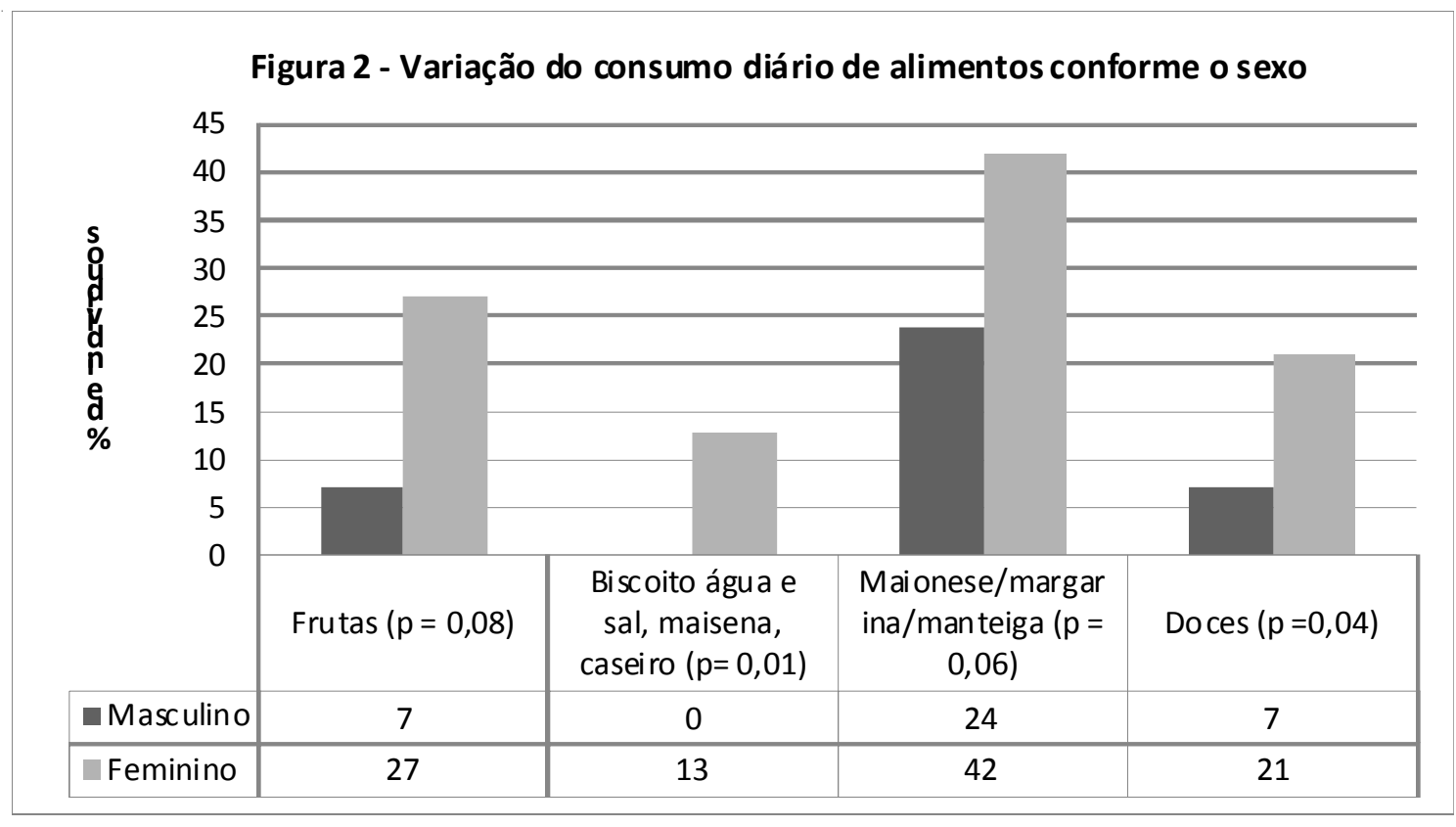

Figura 2 - Variação do consumo diário de alimentos de acordo com o sexo

\section{Discussão}

Este estudo demonstrou que os estudantes universitários apresentaram uma maior frequência diária de ingestão de alimentos considerados de proteção para DCV do que alimentos de risco. Em adição, o ERF calculado demonstrou baixo risco de desenvolvimento de DCV em 10 anos.

Dentro dos alimentos de risco, o consumo de açúcar simples, representado pelo adicionado ao café e aos doces e ao refrigerante se destacou. O café adoçado é comum na dieta de muitos brasileiros e este consumo foi refletido na dieta dos estudantes avaliados. O doce tem se destacado e poderia ser substituído por frutas, principalmente entre as estudantes, que se destacam com relação ao consumo de doces. Já alimentos contendo mais gorduras saturadas e trans aparecem com menor significado no consumo diário, mas ainda assim se faz presente: a maionese/margarina/manteiga se destaca por ser hábito comum de con- 
sumo ao pão junto com café adoçado, refeição de baixo custo e de fácil oferta aos estudantes. Alimentos tipo fast-food também são encontrados provavelmente como substituintes de algumas refeições principais. Esses alimentos são grandes fontes de sódio e sabese que a maior parte do sódio da dieta é proveniente dos compostos sódicos adicionados no processamento dos alimentos ou, em menor escala, do sal de mesa. ${ }^{2}$ Torna-se necessário considerar também que no Brasil, a utilização de gorduras hidrogenadas é ampla e muitas vezes indiscriminada, envolvendo a produção de margarinas, cremes vegetais, pães, biscoitos, batatas fritas, massas, sorvetes, pastéis, bolos, entre outros alimentos. ${ }^{3}$

Os alimentos protetores que apresentaram pequeno consumo diário, como soja, aveia e azeite, foram menos consumidos por serem culturalmente menos comuns ou até mesmo por uma questão financeira dos estudantes, pois são alimentos relativamente mais caros no mercado brasileiro. Tais alimentos também são oferecidos, mas não diariamente aos estudantes pelo refeitório da instituição. $\mathrm{O}$ aumento do consumo de produtos de soja pode implicar na redução do consumo de alimentos ricos em gorduras saturadas e colesterol e, sendo assim, pode exercer um efeito indireto na redução do colesterol sanguíneo..$^{5}$ Já o contrário ocorre com legumes, verduras e frutas que se destacam como os alimentos de proteção de maiores consumo. Pela variedade do grupo de alimentos que o compõe, sua variabilidade de sabores, preços, oferta e acessibilidade fazem mais parte da vida dos estudantes. O que se deve destacar é a variação de consumo com relação ao sexo, as estudantes consomem mais frutas do que os estudantes do sexo masculino, o que pode refletir um fator cultural. A ingestão de flavonoides pela população brasileira é de 60 a 106mg/dia, quantidade obtida principalmente dos vegetais, tomate, alface e laranja, muito embora o café pertença ao grupo de alimentos com maior conteúdo de antioxidantes ${ }^{7}$.

O ERF fornece a probabilidade de um evento cardiovascular nos próximos 10 anos e permite classificá-los em grupos de alto e baixo risco. O presente estudo determinou o ERF em indivíduos supostamente saudáveis. Mesmo que o ERF ainda não te- nha sido validado para indivíduos com idade inferior a 30 anos, a sua utilização nesta população poderia ajudar a estratificar o risco cardiovascular em indivíduos jovens. Os dados obtidos sugerem que o parâmetro que mais influenciou no cálculo do ERF foi a dislipidemia, considerando que os grupos não apresentaram indivíduos diabéticos ou hipertensos. O ERF foi significativamente maior em mulheres jovens em relação aos homens da mesma idade, fato que pode ser explicado pela maior prevalência de dislipidemias observada nas mulheres.

Uma das limitações desse estudo foi o fato de que o mesmo não quantificou as porções dos alimentos, uma vez que determinados alimentos precisam ter um mínimo ou um máximo de consumo para estabelecer sua função de risco ou de proteção para DCV. Outra limitação foi o fato deste estudo não ter separado qual a influência da alimentação feita no restaurante universitário ou não, visto que o cardápio definido pelo referido restaurante limita a escolha alimentar do aluno de graduação, ao contrário, por exemplo, de um universitário que comeria num restaurante no modelo self-service.

\section{Conclusão}

Os dados apontam como hábito alimentar dos indivíduos analisados, um consumo diário de alimentos protetores, como verduras, legumes e frutas, porém consumo insuficiente de peixe, sendo a ação das fibras e antioxidantes os principais fatores protetores. Também indicam um consumo diário de alimentos de risco, principalmente doces, que contribuem com aporte calórico e fonte de carboidratos para formação de triglicerídeos, considerado fator deletério do sistema cardiovascular quando está elevado. O ERF foi maior em mulheres jovens em relação aos homens da mesma idade. No entanto, nenhum dos indivíduos avaliados, supostamente saudáveis, apresentou alto risco para um evento cardiovascular nos próximos anos. Estes dados não excluem a possibilidade de que o resultado individual de ERF possa ser utilizado como uma ferramenta para a conscientização precoce e compreensão sobre prevenção cardiovascular. 


\begin{abstract}
Study Design: Descriptive, observational, cross-sectional.

Aims: The aims of this study were to qualitatively describe the frequency of eating certain foods, as risk and protective for cardiovascular disease (CVD), and determine the Framingham Risk Score (FRS) in supposedly healthy individuals, graduate students from a Brazilian public university.

Methodology: Participants were 97 students, 45 males and 52 females, 18-25 years. After signing the consent form, approved by the Ethics Committee on Research, students completed a questionnaire survey data. We studied food classified as risk and protection, as assessed by its chemical composition tables. The Chi-square test was used when the expected frequencies greater than or equal to 5 . For other parameters we used the Fisher exact test.

Results: Among the foods considered protective stood out the daily intake of vegetables (33\%), greens $(22 \%)$ and fruit (17\%). Among the foods considered at risk are the daily intake of coffee / tea with sugar $(39 \%)$, mayonnaise / margarine / butter (34\%) and sweets (14\%). There was variation in consumption according to sex, for the frequencies of 0, 1, 2 and 4 times a week for food: corn flour / cassava, cornstarch cookie /homemade cookie / water and salt cookie, oats, chicken with skin. There was significant variation in daily consumption between the sexes for food: fruit, sweets, mayonnaise / margarine / butter, cornstarch cookie / homemade cookie / water and salt cookie.

Conclusion: This study showed that college students presented higher frequency of daily intake of foods considered protective for CVD than risk food. In addition, the calculated FRS demonstrated low risk of developing CVD in studied individuals.
\end{abstract}

Key-words: Food Habits. Young Adult. Cardiovascular Diseases.

\section{Referências Bibliográficas}

1. Cervato AM, Mazzilli RN, Martins IS, Marucci MFN. Dieta habitual e fatores de risco para doenças cardiovasculares. Rev Saúde Pública. 1997; 3: 227-35.

2. Teixeira MH, Veiga GV, Sichieri R. Consumo de gordura e hipercolesterolemia em uma amostra probabilística de estudantes de Niterói. Arq Bras Endocrinol Metab. 2007; 1: 65-71.

4. Martin CA, Matshushita M, Souza NE. Ácidos graxos trans: implicações nutricionais e fontes na dieta. Rev Nutr. 2004, 3; 351-9.

3. Rique ABR, Soares EA, Meirelles CM. Nutrição e exercício na prevenção e controle das doenças cardiovasculares. Rev Bras Med Esporte. 2002; 6: 244-54.

5. Esteves EA, Monteiro JBR. Efeitos benéficos das isoflavonas de soja em doenças crônicas. Rev Nutr. 2001, 1: 43-52.

6. Silva MAM, Barcelos MFP, Sousa RV, Lima HM, Falco IR, Lima $A L$ et al. Efeito das fibras dos farelos de trigo e aveia sobre o perfil lipídico no sangue de ratos (Rattusnorvegicus) wistar. Ciênc Agrotec. 2003; 6: 1321-9.

7. Rodrigues HG, Diniz YS, Faine LA, Almeida JA, Fernandes AAH, Novelli LB. Suplementação nutricional com antioxidantes naturais: efeito da rutina na concentração de colesterolHDL. Rev Nutr. 2003; 3: 315-20.

8. Lima FEL, Menezes TN, Tavares MP, Szarfarc SC, Fisberg RM. Ácidos graxos e doenças cardiovasculares: uma revisão. Rev Nutr. 2000;13: 73-80.
9. Sales RL, Costa NMC, Monteiro JBR, Peluzio MCG, Coelho $\mathrm{SB}$, Oliveira CG et al. Efeitos dos óleos de amendoim, açafrão e oliva na composição corporal, metabolismo energético, perfil lipídico e ingestão alimentar de indivíduos eutróficos normolipidêmicos. Rev Nutr. 2005; 18:499-511.

10. Lima FA, Sant'ana AEG, Ataíde TR, Omena CMB, Menezes MES, Vasconcelos SML. Café e saúde humana: um enfoque nas substâncias presentes na bebida relacionadas às doenças cardiovasculares. Rev Nutr. 2010; 6:1063-73.

11. Mahan LK, Escott-Stump S. Krause Alimentos, Nutrição e Dietoterapia, São Paulo: Roca, 2005.

12. Shils ME, Shike M, Ross AC, Caballero B, Cousins RJ. Nutrição Moderna na Saúde e na Doença. 10ª edição. Editora Manole. São Paulo, 2009.

13. Pinheiro ABV. Tabela para Avaliação de Consumo Alimentar em Medidas Caseiras. $5^{a}$ edição. Editora Atheneu. São Paulo, 2004.

14. Franco G. Tabela de Composição Química dos Alimentos. $9^{a}$ edição. Editora Atheneu. São Paulo, 2003.

15. Neumann AICP, Shirassu MM, Fisberg RMA. Consumo de alimentos de risco e proteção para doenças cardiovasculares entre funcionários públicos. Rev. Nutr. 2006; 1:19-28. 